\title{
ON THE GROUPS OF REPEATED GRAPHS
}

\section{ROBERT FRUCHT}

In a recently published paper ${ }^{1}$ Kagno showed that Pappus' graph, consisting of the 9 vertices $A, B, C, D, E, F, G, H, I$, and the 27 arcs $A D, A E, A F, A G, A H, A I, B D, B E, B F, B G, B H, B I, C D, C E, C F$, $C G, C H, C I, D G, D H, D I, E G, E H, E I, F G, F H, F I$, has a group of order 1296 which may be generated by the following set of eight substitutions: $(A B C),(A B),(D E F),(D E),(G H I),(G H),(A D G)(B E H)$ $(C F I),(A D)(B E)(C F) .^{2}$ Kagno's proof of this fact (Theorem 5$)^{1}$ is straightforward, but somewhat lengthy, and it seems of interest to note that this theorem follows at once from a more general and almost self-evident theorem on the groups of repeated graphs, if we apply to Pappus' graph the following lemma, also due to Kagno:" "If $G^{\prime}$ is the complement of $G$, then $G$ and $G^{\prime}$ have the same group."3 Indeed the complement $\Pi^{\prime}$ of Pappus' graph contains the $9 \operatorname{arcs} A B, A C$, $B C, D E, D F, E F, G H, G I, H I$; hence $\Pi^{\prime}$ is not connected, but consists of three triangles (or complete 3-points) $A B C, D E F, G H I$; that is, $\Pi^{\prime}$ is a threefold repeated triangle. To such a repeated graph we can apply the following theorem, which is of interest in itself apart from the use made of it here.

THEOREM. If $G$ is a connected graph of $n$ vertices, having no simple loops, with a group $\mathfrak{S}$ of order $h$, and if $\Gamma$ is the graph consisting of $m$ copies $G_{1}, G_{2}, \cdots, G_{m}$ of the same graph $G$, then the group of $\Gamma$ is Polya's "Gruppenkranz" $\mathfrak{S}_{m}[\mathfrak{S}]$, that is, the group of order $m ! h^{m}$ and degree $m n$, whose substitutions may be described briefly as follows: $:^{4}$ Let

Received by the editors March 19, 1948.

${ }^{1}$ I. N. Kagno, Desargues' and Pappus' graphs and their groups, Amer. J. Math. vol. 69 (1947) pp. 859-862.

${ }^{2}$ It may be remarked that the same group might be generated by other sets of fewer elements, for example, by the following one containing only 3 substitutions: $(A B C)(D E),(A D G)(B E H)(C F I),(A D)(B E)(C F)$.

${ }^{3}$ Here the complement $G^{\prime}$ of a graph $G$ (without loops) is to be defined as follows: Let $I_{q}^{p}=I_{p}^{q}=1$, if and only if the vertices $p$ and $q$ are joined by an arc, otherwise let $I_{q}^{p}=I_{p}^{q}=0$. Now, if for any pair of vertices $p, q$ in $G, I_{q}^{p}=1$, then in $G^{\prime}$ let $I_{q}^{p}=0$, and if $^{q} I_{q}^{p}=0$ in $G$, then in $G^{\prime}$ let $I_{q}^{p}=1$. In other applications of this lemma some difficulty may arise from the fact that the complement of a graph may contain isolated points; for example, the complement of a triangle (or complete 3-point) consists only of three isolated points with no arcs connecting them.

${ }^{4} \mathrm{G}$. Pólya, Kombinatorische Anzahlbestimmungen fïr Gruppen, Graphen und chemische Verbindungen, Acta Math. vol. 68 (1937) p. 178. The same groups have also been studied by other authors. We mention only the following papers:

Wilhelm Specht, Eine Verallgemeinerung der symmetrischen Gruppe, Schriften des 
the vertices of $G_{i}(i=1,2, \cdots, m)$ be denoted by the letters $z_{i 1}, z_{i 2}, \cdots$, $z_{\text {in }}$, and form the rectangular matrix

$$
\left(\begin{array}{cccc}
z_{11} & z_{12} & \cdots & z_{1 n} \\
z_{21} & z_{22} & \cdots & z_{2 n} \\
\cdot & \cdot & \cdot & \cdot \\
z_{m 1} & z_{m 2} & \cdots & z_{m n}
\end{array}\right)
$$

then the substitutions of $\mathfrak{S}_{m}[\mathfrak{S}]$ may be characterized as permutations of the rows of the matrix, followed by permutations of the letters in each row according to some substitution of the group $\mathfrak{S}$.

As the proof is fairly obvious and can readily be supplied by the reader, we shall omit the details. It is evident that the graph $\Gamma$ (consisting of $m$ copies of the connected graph $G$ ) is mapped into itself by the substitutions of the "Gruppenkranz" $\widetilde{S}_{m}[\mathfrak{S}]$; but, since $G$ is a connected graph with group $\mathfrak{S}$, there cannot be substitutions other than those of $\mathfrak{S}_{m}[\mathfrak{S}]$ which map $\Gamma$ into itself.

In the case of the complement $\Pi^{\prime}$ of Pappus' graph we have $m=3, n=3, \mathfrak{S}=\mathfrak{S}_{3}$ (symmetric group on three letters) with $h=6$; the group $\mathfrak{\Im}_{3}\left[\Im_{3}\right]$ is of order $3 ! 6^{3}=1296$, and is obviously the same as that obtained by Kagno.

As another example let us consider the graph called $H_{18}$ by Kagno in a previous paper, ${ }^{5}$ that is, the graph consisting of six vertices $a, b, c, d, e, f$, and the nine arcs $a d, a e, a f, b d, b e, b f, c d, c e, c f$. Its complement, having the arcs $a b, a c, b c, d e, d f, e f$, consists of two triangles; hence its group is the "Gruppenkranz" $\mathfrak{S}_{2}\left[\mathfrak{\Im}_{3}\right]$ of order $2 ! 6^{2}=72$. (In Kagno's paper ${ }^{5}$-cf. his Theorem 3.17 -this group is called $\mathfrak{S}_{34}$ $\equiv(a b c d e f)_{72}$. $)$

A third example ${ }^{6}$ is furnished by the graph formed by the vertices and edges of the regular octahedron; its group is $\Im_{3}\left[\Im_{2}\right]$ of order $3 ! 2^{3}=48$. (In Kagno's paper ${ }^{5}$ this is the graph $H_{5}$ with the group $\mathfrak{S}_{32} \equiv(a b c d e f)_{48} ;$ see his Theorem 3.4 in the corrected form.)

Mathematischen Seminars und des Instituts für Angewandte Mathematik der Universität Berlin, vol. 1, 1931.

Oystein Ore, Theory of monomial groups, Trans. Amer. Math. Soc. vol. 51 (1942) pp. 15-64.

R. Frucht, Coronas de grupos y sus subgrupos, con una aplicacion a los determinantes, Union Matematica Argentina, Publicacion No. 24, 1942.

5 I. N. Kagno, Linear graphs of degree $\leqq 6$ and their groups, Amer. J. Math. vol. 68 (1946) pp. 505-520. See also Corrections on page 872 of vol. 69 (1947) of the same Journal.

6 Polya, loc. cit. p. 214. 
Finally it may be remarked that a theorem analogous to our theorem would hold for the automorphism groups of "connected systems" other than graphs, for example, for connected partially ordered systems, for certain functions of several variables, and so on. (Thus the group $\Im_{3}\left[\Im_{3}\right]$ might also be characterized as the largest permutation group on 9 variables $x_{1}, x_{2}, \cdots, x_{9}$, under which the function $x_{1} x_{2} x_{3}$ $+x_{4} x_{5} x_{6}+x_{7} x_{8} x_{9}$ is invariant.)

Technical University Santa Maria, Valparaiso, Chile 